\title{
The Levenberg-Marquardt Method and Its Modified Versions for Solving Nonlinear Equations with Application to the Inverse Gravimetry Problem
}

\author{
V. V. $\operatorname{Vasin}^{1,2}$ and G. Ya. Perestoronina ${ }^{1}$ \\ Received October 19, 2010
}

\begin{abstract}
The Levenberg-Marquardt method and its modified versions are studied. Under some local conditions on the operator (in a neighborhood of a solution), strong and weak convergence of iterations is established with the solution error monotonically decreasing. The conditions are shown to be true for one class of nonlinear integral equations, in particular, for the structural gravimetry problem. Results of model numerical experiments for the inverse nonlinear gravimetry problem are presented.

Keywords: Levenberg-Marquardt method, modified process, a priori information, inverse gravimetry problem.
\end{abstract}

DOI:10.1134/S0081543813020144

\section{INTRODUCTION}

The Levenberg-Marquardt method belongs to the class of iteratively regularized Gauss-Newton methods. This method is successfully applied for solving various ill-posed inverse problems of filtration, geophysics, and atmospheric sounding (see $[1,2,5-7]$ ). For the nonlinear operator equation

$$
A(u)=f
$$

with a Fréchet differentiable operator $A$ acting on a pair of Hilbert spaces $U$ and $F$, iterations in this method are constructed as follows:

$$
u^{k+1}=u^{k}-\left[A^{\prime}\left(u^{k}\right)^{*} A^{\prime}\left(u^{k}\right)+\alpha_{k} I\right]^{-1} A^{\prime}\left(u^{k}\right)^{*}\left(A\left(u^{k}\right)-f\right),
$$

where $\alpha_{k}$ is some positive sequence of control parameters.

The theoretical investigation of process $(1.2)$ can be found in $[1,2,7]$. Proving the convergence of iterations, one has to impose certain conditions on the choice of the parameter $\alpha_{k}$. For example, the following principle of the parameter choice is proposed in [7]: the parameter $\alpha=\alpha_{k}$ must be such that

$$
\left\|f_{\delta}-A\left(u^{k}\right)-A^{\prime}\left(u^{k}\right)\left(u^{k+1}(\alpha)-u^{k}\right)\right\|=q\left\|f_{\delta}-A\left(u^{k}\right)\right\|,
$$

\footnotetext{
${ }^{1}$ Institute of Mathematics and Mechanics, Ural Branch of the Russian Academy of Sciences, ul. S. Kovalevskoi 16, Yekaterinburg, 620990 Russia

emails: vasin@imm.uran.ru,pgja@imm.uran.ru

${ }^{2}$ Institute of Mathematics and Computer Science, Ural Federal University, pr. Lenina 51, Yekaterinburg, 620000 Russia
} 
where $0<q<1$ and $\left\|f-f_{\delta}\right\| \leq \delta$. The existence of a unique solution $\alpha_{k}$ is guaranteed if

$$
\left\|f_{\delta}-A\left(u^{k}\right)-A^{\prime}\left(u^{k}\right)\left(z-u^{k}\right)\right\| \leq \frac{q}{\gamma}\left\|f_{\delta}-A\left(u^{k}\right)\right\|,
$$

where $\gamma>1$ and $z$ is a solution of equation (1.1) with minimal norm; its existence is assumed in some neighborhood $S_{\rho}\left(u^{0}\right)=\left\{u:\left\|u-u^{0}\right\| \leq \rho\right\}$. The strong convergence of iterations to the normal solution of equation (1.1) as $\delta \rightarrow 0$ is proved under the assumptions that

$$
\left\|A(u)-A(\tilde{u})-A^{\prime}(u)(u-\tilde{u})\right\| \leq c\|A(u)-A(\tilde{u})\| \quad \forall u, \tilde{u} \in S_{2 \rho}\left(u^{0}\right) \subset D(A),
$$

and the iteration number $k(\delta)$ in the stopping rule is chosen according to the residual principle

$$
\left\|f_{\delta}-A\left(u^{k(\delta)}\right)\right\| \leq \tau \delta<\left\|f_{\delta}-A\left(u^{k}\right)\right\|, \quad 0 \leq k<k(\delta) .
$$

In $[2$, Sect. 6], the variant of the Levenberg-Marquardt method with additional parameter $\beta>0$

$$
u^{k+1}=u^{k}-\beta\left[A^{\prime}\left(u^{k}\right)^{*} A^{\prime}\left(u^{k}\right)+\alpha_{k} I\right]^{-1} A^{\prime}\left(u^{k}\right)^{*}\left(A\left(u^{k}\right)-f\right) \equiv T_{k}\left(u^{k}\right)
$$

is considered, and a theorem on its weak convergence is proved under the conditions that

$$
\|A(u)-f\|^{2} \leq \varkappa\left\langle A^{\prime}(u)(u-z), A(u)-f\right\rangle,
$$

the operator $S(u)=A^{\prime}(u)^{*}(A(u)-f)$ is weakly-strongly closed, and $0<\beta<2 \alpha /\left(\varkappa N_{1}^{2}\right)$, where $\alpha_{k} \geq \alpha>0,\left\|A^{\prime}(u)\right\| \leq N$, and $u \in S_{\rho}(z)$. Actually, the proof uses a condition on the choice of the parameter $\alpha_{k}$ guaranteeing the inequalities

$$
\left\|u^{k+1}-z\right\|_{k+1} \leq\left\|u^{k+1}-z\right\|_{k}
$$

where $\|u\|_{k}^{2}=\left\langle B_{k} u, u\right\rangle, B_{k}=A^{\prime}\left(u^{k}\right)^{*} A^{\prime}\left(u^{k}\right)+\alpha_{k} I$, and $z$ is a solution of equation (1.1).

Note that the choice of the parameter $\alpha_{k}$ according to (1.3), (1.4), (1.7), or (1.8) is not constructive, since these relations contain an exact solution which is unknown for real data. However, we show that condition (1.7) holds in a sufficiently small neighborhood of the required solution for some class of nonlinear integral equations, in particular, for the gravimetry equation.

In the investigation of the weak convergence of process (1.6), it is sufficient to require that condition (1.7) hold only at iterative points, i.e., for $u=u^{k}$. In this case, we can also consider condition (1.7) as a condition on the choice of the regularization parameter similarly to condition (1.4), which is stronger than (1.7).

In the present paper, in the investigation of the convergence of method (1.6), we introduce the following local condition, which is somewhat different from (1.7):

$$
\|A(u)-f\|^{2} \leq \bar{\varkappa}\left\langle B_{k}^{-1}(u) S(u), u-z\right\rangle, \quad \bar{\varkappa}>0,
$$

where $B_{k}(u)=A^{\prime}(u)^{*} A^{\prime}(u)+\alpha_{k} I$. We establish the weak convergence of the method.

To obtain the strong convergence of iterations, we pass from (1.6) to a process (see (2.7)) with an additional operation of metric projection onto a convex compact set $Q$ containing, by assumption, the required solution. Method (1.6) and its variant with projection are studied in Section 2.

Along with process (1.6), we consider the modified Levenberg-Marquardt method

$$
u^{k+1}=u^{k}-\gamma\left[A^{\prime}\left(u^{0}\right)^{*} A^{\prime}\left(u^{0}\right)+\alpha_{k} I\right]^{-1} A^{\prime}\left(u^{k}\right)^{*}\left(A\left(u^{k}\right)-f\right) \equiv T_{0}\left(u^{k}\right),
$$


where, in contrast to (1.6), the parameter $\alpha_{k}=\alpha$ is fixed and $u^{k}$ in the inverted operator is replaced by a constant element $u^{0}$, which plays the role of the initial approximation. The weak convergence of process (1.10) can be established under local condition (1.7), which, as mentioned above, is valid for one important class of integral equations. Questions of the convergence of method (1.10) and its modification are contained in Section 3. In Section 4, we give a comparative analysis of the numerical efficiency of iterative processes under investigation.

\section{CONVERGENCE OF THE LEVENBERG-MARQUARDT METHOD}

2.1. Consider process (1.6), where $\beta>0$ and the sequence of parameters $\alpha_{k}$ is such that $\overline{\bar{\alpha}} \geq \alpha_{k} \geq \bar{\alpha}>0$. Assume that $\sup \left\{\left\|A^{\prime}(u)\right\|: u \in S_{\rho}(z)\right\} \leq N_{1}<\infty$ in some neighborhood $S_{\rho}(z)$ of the required solution $z$, and the ball $S_{\rho}(z)=\{u$ : $\|u-z\| \leq \rho\}$ contains no other solutions.

Theorem 2.1. Suppose that $A$ is a Fréchet differentiable and weakly-strongly closed operator acting on a pair of Hilbert spaces $U$ and $F$; i.e.,

$$
u^{k} \rightarrow \bar{u} \quad(\text { weakly }), \quad A\left(u^{k}\right) \rightarrow \bar{f} \Rightarrow \bar{u} \in D(A), \quad A(\bar{u})=\bar{f} .
$$

Assume that local condition (1.9) holds.

Then, process (1.6) has the following properties for $\beta<\frac{2 \bar{\alpha}}{\bar{\varkappa} N_{1}^{2}}$ :

(1) $u^{k} \rightarrow z($ weakly);

(2) either $\left\|u^{k+1}-z\right\|<\left\|u^{k}-z\right\|$ for any $k$ or $u^{k}$ is stationary for $k \geq k_{0}$ and $u^{k_{0}}=z$;

(3) $\sum_{k=0}^{\infty}\left\|u^{k+1}-u^{k}\right\|^{2} \leq \frac{\left\|u^{0}-z\right\|^{2}}{\nu}, \quad \nu=\frac{2 \bar{\alpha}^{2}}{\bar{\varkappa} N_{1}^{2} \beta}-1$;

(4) $\lim _{k \rightarrow \infty}\left\|A\left(u^{k}\right)-f\right\|=0$.

Proof. Let us check that the inequality

$$
\left\|T_{k}(u)-z\right\|^{2}-\|u-z\|^{2}+\nu\left\|u-T_{k}(u)\right\|^{2} \leq 0,
$$

i.e., the condition of pseudocontractivity of $T_{k}$, holds for some $\nu>0$. By (1.6), the inequality is transformed to the form

$$
-\frac{2}{(1+\nu) \beta}\left\langle B_{k}^{-1} S(u), u-z\right\rangle+\left\|B_{k}^{-1} S(u)\right\|^{2} \leq 0 .
$$

Since $\left\|B_{k}^{-1}\right\| \leq 1 / \bar{\alpha}$ and $\left\|A^{\prime}\left(u^{k}\right)\right\| \leq N_{1}$, if the inequality

$$
-\frac{2}{(1+\nu) \beta}\left\langle B_{k}^{-1} S(u), u^{k}-z\right\rangle+\frac{N_{1}^{2}}{\alpha^{2}}\|A(u)-f\|^{2} \leq 0
$$

holds, then relation (2.3) is also valid. Combining (2.4) and (1.9), we find that, if

$$
\beta<\frac{2 \bar{\alpha}^{2}}{\bar{\varkappa} N_{1}^{2}}
$$

relation (2.2) holds for

$$
\nu \leq \frac{2 \bar{\alpha}^{2}}{\bar{\varkappa} N_{1}^{2} \beta}-1
$$


Inequality (2.2) with $u=u^{k}$ implies that

$$
\begin{gathered}
\left\|u^{k+1}-z\right\| \leq\left\|u^{k}-z\right\|, \quad \lim _{k \rightarrow \infty}\left\|u^{k}-z\right\|=d(z) \geq 0, \\
u^{k+1}-u^{k}=-\beta B_{k}^{-1} S\left(u^{k}\right) \rightarrow 0 .
\end{gathered}
$$

Since $\left\|B_{k}\right\| \leq N_{1}^{2}+\overline{\bar{\alpha}}$, relation (2.6) implies the convergence $S\left(u^{k}\right) \rightarrow 0$, and (2.5) implies the existence of a weakly convergent subsequence $u^{k_{i}} \rightarrow \bar{u}$ (weakly). By the relations obtained and (1.9), we have $A\left(u^{k_{i}}\right) \rightarrow f$. In view of the weak-strong closedness of $A$ and relation (2.5), we get $\bar{u} \in D(A) \cap S_{\rho}(z)$ and $A(\bar{u})=f$; i.e., $\bar{u}=z$ is a solution of equation (1.1). Thus, $\bar{u}=z$ is the unique weak limit point of the sequence $\left\{u^{k}\right\}$; this implies properties (1) and (4).

Properties (2) and (3) are proved as in the general case for pseudocontractive operators [3].

Theorem 2.2. Suppose that the operator A satisfies the assumptions of Theorem 2.1 with local condition (1.7) instead of condition (1.9). Suppose also that relation (1.4) holds at iterative points of process (1.2) and the parameter $\alpha_{k}$ is chosen according to rule (1.3) for $f_{\delta}=f$; moreover, the estimate $\alpha_{k} \geq \bar{\alpha}>0$ is valid. Then, the sequence $\left\{u^{k}\right\}$ generated by process (1.2) satisfies properties (1)-(4) from Theorem 2.1.

Proof. Under the assumptions of the theorem, the inequality

$$
\left\|u^{k+1}-z\right\|^{2} \leq\left\|u^{k}-z\right\|^{2}-\left\|u^{k+1}-u^{k}\right\|^{2}
$$

is proved in [1, Proposition 4.1]. This inequality can be written in the form

$$
\left\|T_{k}\left(u^{k}\right)-z\right\|^{2} \leq\left\|u^{k}-z\right\|^{2}-\left\|T_{k}\left(u^{k}\right)-u^{k}\right\|^{2},
$$

which yields (2.5) and (2.6) for $\beta=1$. The proof is completed similarly to the proof of Theorem 2.1.

2.2. Assume that there is an additional a priori information that the required solution $z$ belongs to a convex compact or boundedly compact set $Q$ (see [4]). Consider the iterative process

$$
v^{k+1}=P_{Q}\left(T_{k}\left(v^{k}\right)\right) \equiv F_{k}\left(v^{k}\right), \quad v^{0} \in S_{\rho}(z),
$$

where $P_{Q}$ is the metric projection onto $Q$, and $T_{k}$ is the step operator in method (1.6).

Theorem 2.3. Suppose that the assumptions of Theorem 2.1 hold except for the weak-strong closedness of the operator A. Then, process (2.7) generates a sequence $\left\{v^{k}\right\}$ strongly convergent to a solution $z$ of equation (1.1).

Proof. Since $P_{Q}$ and $T_{k}$ are pseudocontractive operators, i.e., relation (2.2) holds for each of them with some $\nu>0$, their superposition $F_{k}=P_{Q} T_{k}$ is a pseudocontractive mapping (see [2]), for which $z$ is the unique fixed point.

Successively using the pseudocontractivity property, we obtain

$$
\begin{gathered}
\left\|v^{k+1}-z\right\|=\left\|P_{Q}\left(T^{k}\left(v^{k}\right)\right)-z\right\|^{2} \leq\left\|T_{k}\left(v^{k}\right)-z\right\|^{2}-\left\|T_{k}\left(v^{k}\right)-P_{Q}\left(T_{k}\left(v^{k}\right)\right)\right\|^{2} \\
\leq\left\|v^{k}-z\right\|^{2}-\nu\left\|v^{k}-T_{k}\left(v^{k}\right)\right\|^{2}-\left\|T_{k}\left(v^{k}\right)-P_{Q}\left(T_{k}\left(v^{k}\right)\right)\right\|^{2},
\end{gathered}
$$

which implies

$$
\begin{gathered}
\left\|v^{k+1}-z\right\| \leq\left\|v^{k}-z\right\|, \quad\left\|v^{k}\right\| \leq c, \quad c \text { is a constant } \\
\lim _{k \rightarrow \infty}\left\|v^{k+1}-z\right\|=d(z) \geq 0, \quad v^{k}-T_{k}\left(v^{k}\right) \rightarrow 0, \quad T_{k}\left(v^{k}\right)-P_{Q}\left(T_{k}\left(v^{k}\right)\right) \rightarrow 0 .
\end{gathered}
$$


Since $Q$ is a compact set and $v^{k} \in Q$, there exists a convergent subsequence

$$
v^{k_{i}} \rightarrow \bar{v} \in Q \cap S_{\rho}(z) .
$$

It follows from relations $(2.8)$ and (2.9) that

$$
v^{k_{i}}-T_{k_{i}}\left(v^{k_{i}}\right)=\beta B_{k_{i}}^{-1}\left(v^{k_{i}}\right) S\left(v^{k_{i}}\right) \rightarrow 0 .
$$

Combining (2.10) and (1.9) for $u=v^{k_{i}}$, we arrive at

$$
A\left(v^{k_{i}}\right)-f \rightarrow 0 \text {. }
$$

Since $A$ is a continuous operator, which follows from its differentiability, we obtain from (2.9) and (2.11) that $A(\bar{v})=f$; i.e., $\bar{v}$ coincides with the required solution $z$. By the uniqueness of a solution on the set $Q \cap S_{\rho}(z)$, we have the convergence of the whole sequence $\left\{v^{k}\right\}$ to the solution $z$.

\section{A MODIFIED LEVENBERG-MARQUARDT METHOD}

3.1. Now, consider modified Levenberg-Marquardt process (1.10), where, in contrast to the main process, the derivative $A^{\prime}(u)$ in the inverted operator is calculated at a fixed point $u_{0}$, which is considered as the initial approximation.

Let us introduce a new Hilbert norm in the space $U$ :

$$
\|u\|_{0}^{2}=\left\langle B_{0} u, u\right\rangle, \quad B_{0}=A^{\prime}\left(u_{0}\right)^{*} A^{\prime}\left(u_{0}\right)+\alpha I, \quad \alpha>0 .
$$

Denote by $U_{0}$ the Hilbert space with this norm.

Theorem 3.1. Let a Fréchet differentiable weakly-strongly closed operator (see (2.1)) satisfy local condition (1.7) in some neighborhood of the required solution $z$.

Then, the following properties are valid for process (1.10) for $0<\gamma<\frac{2 \alpha}{\varkappa N_{1}^{2}}$ and $u^{0} \in S_{\rho}(z)$ :

(1) $u^{k} \rightarrow z$ (weakly) in the space $U$;

(2) either $\left\|u^{k+1}-z\right\|_{0}<\left\|u^{k}-z\right\|_{0}$ for any $k$ or $u^{k}$ is stationary for $k \geq k_{0}$; moreover, $u^{k_{0}}=z$;

(3) $\sum_{k=0}^{\infty}\left\|u^{k+1}-u^{k}\right\|_{0}^{2} \leq \frac{\left\|u^{0}-z\right\|_{0}^{2}}{\nu}$;

(4) $\lim _{k \rightarrow \infty}\left\|A\left(u^{k}\right)-f\right\|=0$.

Proof. Let us check that, in the new Hilbert norm defined by relations (3.1), the step operator

$$
T_{0}(u)=u-\gamma B_{0}^{-1} S(u), \quad S(u)=A^{\prime}(u)^{*}(A(u)-f),
$$

is a pseudocontractive operator; i.e., the following inequality holds for some $\nu>0$ :

$$
\left\|T_{0}(u)-z\right\|_{0}^{2}-\|u-z\|_{0}^{2}+\nu\left\|u-T_{0}(u)\right\|_{0}^{2} \leq 0 .
$$

Using the expression for the operator $T_{0}$, we obtain from (3.2) the inequality

$$
-\frac{2}{(1+\nu) \gamma}\langle u-z, S(u)\rangle+\left\langle S(u), B_{0}^{-1} S(u)\right\rangle \leq 0,
$$

which holds a priori if

$$
-\frac{2}{(1+\nu) \gamma}\langle u-z, S(u)\rangle+\frac{N_{1}^{2}}{\alpha}\|A(u)-f\|^{2} \leq 0 .
$$


In view of (1.7), inequality (3.3) holds if

$$
\frac{2 \alpha}{(1+\nu) \gamma N_{1}^{2}} \geq \varkappa
$$

which yields the condition on the step parameter

$$
\gamma<\frac{2 \alpha}{\varkappa N_{1}^{2}}
$$

moreover, the parameter $\nu$ in (3.2) can be chosen in accordance with the condition

$$
\nu \leq \frac{2 \alpha}{\varkappa \gamma N_{1}^{2}}-1
$$

Inequality (3.2) with $u=u^{k}$ implies the relations

$$
\begin{gathered}
\lim _{k \rightarrow \infty}\left\|u^{k}-z\right\|_{0}=d(z), \quad\left\|u^{k}\right\|_{0} \leq c, \quad c \text { is a constant } \\
\lim _{k \rightarrow \infty}\left\|T\left(u^{k}\right)-u^{k}\right\|_{0}=\gamma\left\|B_{0}^{-1} S\left(u^{k}\right)\right\|_{0}=0 .
\end{gathered}
$$

Since the obvious inequalities

$$
\alpha\|u\|^{2} \leq\|u\|_{0}^{2} \leq\left(N_{1}^{2}+\alpha\right)\|u\|^{2}
$$

hold, which mean the equivalence of the norms, (3.4) implies the boundedness of the sequence $\left\{u^{k}\right\}$ in the original space $U$.

Therefore, in the space $U$, there exists a weakly convergent subsequence

$$
u^{k_{i}} \rightarrow \bar{u} \quad(\text { weakly), } \quad i \rightarrow \infty .
$$

Since the norms are equivalent and the operator $B_{0}$ is continuous, (3.5) implies

$$
S\left(u^{k}\right) \rightarrow 0, \quad i \rightarrow \infty .
$$

From condition (1.7) and relations (3.6) and (3.7), we find that

$$
A\left(u^{k_{i}}\right)-f \rightarrow 0, \quad i \rightarrow \infty .
$$

Taking into account the fact that $A$ is a weakly-strongly closed operator, from (3.6) and (3.8) we have $\bar{u} \in D(A)$ and $A(\bar{u})=f$. By the monotonicity $\left\|u^{k+1}-z\right\|_{0} \leq\left\|u^{k}-z\right\|_{0}$, which follows from (3.2) for $u=u^{k}$, and by the condition $u^{0} \in S_{\rho}(z)=\left\{u:\|u-z\|_{0} \leq \rho\right\}$, all iterative points $u^{k}$ belong to $S_{\rho}(z)$. In view of the weak closedness of $S_{\rho}(z)$, the limit point $\bar{u}$ from (3.6) also belongs to $S_{\rho}(z)$. Since we assume that there are no other solutions in $S_{\rho}(z)$ except for $z$, we have $\bar{u}=z$.

The proof is completed by the usual scheme for pseudocontractive operators (see [2,3]).

3.2. As in the case of the main process, assume that there is a priori information about the existence of a convex compact or boundedly compact subset $Q \subset U$ to which the required solution $z$ belongs. Denote by $P_{Q}$ the metric projection in $U_{0}$. It is known that $P_{Q}$ is a single-valued continuous pseudocontractive mapping (see, e.g., [3]). Now, we can formulate an analog of Theorem 2.3 for the iterative process

$$
w^{k+1}=P_{Q}\left(T_{0}\left(w^{k}\right)\right) \equiv F_{0}\left(w^{k}\right), \quad w^{0} \in S_{\rho}(z),
$$


where $T_{0}$ is the step operator in method (1.10).

Theorem 3.2. Suppose that the assumptions of Theorem 3.1 hold except for the weak-strong closedness of the operator $A$. Then, process (3.9) generates a sequence $\left\{w^{k}\right\}$ that strongly converges to a solution $z$ of equation (1.1).

For the proof, we can use the property of pseudocontractivity of the operator $F_{0}=P_{Q} T_{0}$ in the space $U_{0}$, the equivalence of norms in the spaces $U$ and $U_{0}$, and arguments similar to the proof of Theorem 2.3.

Remark 3.3. The passage from methods (1.6) and (1.10) to processes (2.7) and (3.9) with projection onto an a priori set allows us to eliminate the condition of the weak-strong closedness of $A$, which is difficult to verify, and obtain the strong convergence of iterations for exact data.

Remark 3.4. We should note that, in a more general situation, when the a priori set $Q$ does not satisfy the condition of compactness, it seems that we cannot guarantee (without additional conditions) the strong convergence of iterative processes (2.7) and (3.9). However, for a convex closed set $Q$, we can obtain weak convergence by using a pseudocontractive mapping instead of the projection $P_{Q}$; this mapping, in contrast to the metric projection, is formed constructively for a wide class of constraints. For example, if

$$
Q=\left\{u: g_{i}(u) \leq 0, \quad i=1,2, \ldots, m\right\},
$$

where $g_{i}$ are differentiable functionals whose gradients are bounded operators, then

$$
P_{Q}(u)=u-\lambda d(u) \frac{e(u)}{\|e(u)\|},
$$

where $d(u)=\sum_{i=1}^{m} c_{i}\left[f^{+}(u)\right]^{2}$ and $e(u)=\nabla d(u)$, is a pseudocontractive mapping (see [2]); hence, processes (2.7) and (3.9) generate iterative sequences weakly convergent to the solution (see [3]).

Remark 3.5. In the above theorems, the convergence of the iterative processes requires local condition (1.7) (Theorems 2.2, 3.1, and 3.2) or condition (1.9) (Theorems 2.1 and 2.3). In [8], it is established that condition (1.7) holds for the integral equation

$$
A[u](x) \equiv \int_{\Pi} K\left(x, x^{\prime}, u\left(x^{\prime}\right)\right) d x^{\prime}=f(x)
$$

under a requirement on the kernel $K\left(x, x^{\prime}, u\right)$ of the form

$$
K_{2} \geq\left|K_{n}^{\prime}\left(x, x^{\prime}, z\right)\right| \geq K_{1}>0, \quad\left|K_{u}^{\prime}\left(x, x^{\prime}, u\right)-K_{u}^{\prime}\left(x, x^{\prime}, z\right)\right| \leq L|u-z|
$$

in a sufficiently small neighborhood $S_{\rho}(z)\left(\rho<K_{1} / 2 L\right)$ of the required solution $z$; here, $x, x^{\prime} \in \Pi \subset \mathbb{R}^{n}$ and $z, u \in M \cap S_{\rho}(z)$, where $M$ is a convex closed subset. Moreover, if the set $M$ is given in the form $M=\left\{u: 0 \leq m_{0} \leq u(x) \leq m_{1}\right\}$, then condition (1.7) holds for the gravimetry equation.

In view of these properties, in order to obtain the convergence of iterations for the gravimetry equation (see (4.2)), it is sufficient to pass from iterative scheme (3.9) to the process

$$
w^{k+1}=P_{Q}\left(T_{0}\left(P_{M}\left(w^{k}\right)\right)\right) .
$$

The introduction of an additional operation of projection $P_{M}$ is connected with the fact that local condition (1.7) for the gravimetry operator (see (4.2)) can be established only on the set $S_{\rho}(z) \cap M$. 
3.3. Let the right-hand side of (1.1) be given with error: $\left\|f-f_{\delta}\right\| \leq \delta$. For iterative processes (2.7) and (3.9), the stopping rule based on the residual principle means that $k(\delta)$ is chosen in accordance with (1.5) for some $\tau>1$. If, for a continuous one-to-one operator $A$, such a number exists for $0<\delta \leq \delta_{0}$, then these processes generate regularizing algorithms. This follows from the estimate

$$
\left\|A\left(u^{k(\delta)}\right)-A(z)\right\| \leq\left\|A\left(u^{k(\delta)}\right)-f_{\delta}\right\|+\left\|f_{\delta}-f\right\| \leq(1+\tau) \delta
$$

and the Hausdorff-Tikhonov lemma about the continuity of the inverse operator on a compact set.

\section{NUMERICAL EXPERIMENTS}

To verify the effectiveness of iterative processes (1.6) and (1.10), we conducted numerical experiments on the reconstruction of the model solution

$$
z\left(x_{1}, x_{2}\right)=5+\frac{4}{\pi} \arctan \left(\frac{2}{5 x_{1}-10}\right)+\sin \left(\frac{\pi}{30 x_{2}}\right),
$$

taken from paper [8], for the gravimetry equation

$$
\begin{aligned}
& A[u](x) \equiv \Gamma \Delta \sigma\left\{\int_{\Pi} \frac{d x^{\prime}}{\left[\left(x_{1}-x_{1}^{\prime}\right)^{2}+\left(x_{2}-x_{2}^{\prime}\right)^{2}+H^{2}\right]^{1 / 2}}\right. \\
& \left.-\int_{\Pi} \frac{d x^{\prime}}{\left[\left(x_{1}-x_{1}^{\prime}\right)^{2}+\left(x_{2}-x_{2}^{\prime}\right)^{2}+u^{2}\left(x^{\prime}\right)\right]^{1 / 2}}\right\}=f(x) ;
\end{aligned}
$$

here, $x=\left(x_{1}, x_{2}\right), d x^{\prime}=d x_{1}^{\prime} d x_{2}^{\prime}$, the density jump $\Delta \sigma=0.5 \mathrm{~g} / \mathrm{cm}^{3}$, and $H=5 \mathrm{~km}$. The model anomalous field was calculated for $z\left(x_{1}, x_{2}\right)$ from (4.1) for the domain $\Pi=\left\{0 \leq x_{1} \leq 50 \mathrm{~km}\right.$, $\left.0 \leq x_{2} \leq 60 \mathrm{~km}\right\}$ and was considered as exact calculation data.

Integral equation (4.2) was reduced to a system of nonlinear equations by means of a twodimensional analog of the rectangle formula on the $100 \times 30$ grid with uniform step in each variable.

When process (1.6) was used with initial approximation $u^{0}=6 \mathrm{~km}$ and parameters $\beta=1$ and $\alpha=1$, the residual decreased by a factor of 3000 after 30 iterations, and the relative mean-square error was $\varepsilon_{1}^{30}=\left\|u^{30}-z\right\| /\|z\| \simeq 0.6 \times 10^{-2}$ for the solution and $\varepsilon_{2}^{30}=\left\|A\left(u^{30}\right)-f\right\| /\|f\|=0.4 \times 10^{-3}$ for the residual. The values

$$
\Delta_{1}^{k}=\left\|u^{k}-z\right\|, \quad \Delta_{2}^{k}=\left\|A\left(u^{k}\right)-f\right\|
$$

decreased monotonically. The monotone decrease of the error $\Delta_{1}^{k}$ is due to the fact that, at the iterative points $(2 \leq k \leq 30)$, local condition (1.9) holds for $\bar{\varkappa} \geq 3.07$ and condition (1.7) holds for $\varkappa \geq 1.05$. This guarantees the validity of relation (2.2) for $u=u^{k}$ and, consequently, $\left\|u^{k+1}-z\right\|^{2}<\left\|u^{k}-z\right\|$ (see Theorems 2.1 and 2.2).

For the disturbed right-hand side with error about $5 \%$, the relative error of the solution was $\tilde{\varepsilon}_{1}^{30}=\left\|\tilde{u}^{30}-z\right\| /\|z\| \simeq 0.02$.

We performed 60 iterations of modified process (1.10) with parameters $\gamma=1$ and $\alpha_{k}=10$ for the same model solution and initial approximation $u^{0}=\bar{u}^{2}$, where $\bar{u}^{2}$ is the vector obtained after two iterations of the main process. For undisturbed data, the relative error was $\varepsilon_{1}^{60}=\left\|u^{60}-z\right\| /\|z\| \simeq$ 0.01 for the solution and $\varepsilon_{2}^{60}=\left\|A\left(u^{60}\right)-f\right\| /\|f\| \simeq 0.9 \times 10^{-2}$ for the residual.

As in the case of the main process, the error $\Delta_{1}^{k}=\left\|u^{k}-z\right\|_{0}(k=2,3, \ldots, 60)$ monotonically decreased, since relation (3.2) holds at the iterative points. In comparison with the main process, 
the modified process converged more slowly. For example, the main process gave the same accuracy for the solution and for the residual after the 4th iteration as the modified method after the 60th iteration. In addition, we had to choose the initial point $u^{0}$ in a neighborhood of the solution of smaller radius. For example, for $u^{0}=6$, the main process, as noted above, converges and the modified process diverges.

\section{CONCLUSIONS}

We proposed a modified version of the Levenberg-Marquardt method, in which an element is fixed in the inverted operator. Theorems about the weak and strong convergence are formulated for the Levenberg-Marquardt method and its modifications under local conditions in a neighborhood of the required solution. Local condition (1.7) holds for one class of nonlinear integral equations, in particular, for the inverse gravimetry problem. Another condition for the main process in model calculations can be verified at iterative points, which guarantees the monotone decrease of the solution error. Numerical experiments showed that, for the same number of iterations, the main Levenberg-Marquardt process essentially exceeds the modified version in accuracy and does not require strict conditions on the initial approximation. However, the modified version of the method is simpler for implementation and essentially more economical with respect to computing time, since it does not require a recalculation of the inverted operator. The same tendency is preserved for the comparison of the Levenberg-Marquardt method with processes of gradient type such as the methods of minimal error and gradient descent: the time required to obtain the same accuracy in the numerical implementation of the Levenberg-Marquardt method is by a factor of a hundred larger than in gradient processes (see [8]).

The numerical experiments showed the stable behavior of iterations; hence, there was no necessity to use the operations of projection $P_{Q}$ and $P_{M}$ on a priori sets.

\section{ACKNOWLEDGMENTS}

This work was supported by the Russian Foundation for Basic Research (project no. 09-0100053) and by the Ural Branch of the Russian Academy of Sciences within Interdisciplinary project no. 09-M-15-2001.

\section{REFERENCES}

1. B. Kaltenbacher, A. Neubauer, and O. Scherzer, Iterative Regularization Methods for Nonlinear Ill-Posed Problems (Walter de Gruyter, Berlin, 2008).

2. V. V. Vasin and I. I. Eremin, Operators and Iterative Processes of Fejér Type: Theory and Applications (Walter de Gruyter, Berlin, 2009).

3. V. V. Vasin and A. L. Ageev, Ill-Posed Problems with A Priori Information (VSP, Utrecht, 1995).

4. V. K. Ivanov, V. V. Vasin, and V. P. Tanana, Theory of Linear Ill-Posed Problems and Its Applications (Nauka, Moscow, 1978; VSP, Utrecht, 2002).

5. V. V. Vasin, G. Ya. Perestoronina, I. L. Prutkin, and L. Yu. Timerkhanova, Mat. Model. 15 (2), 69 (2003).

6. A. L. Ageev, T. V. Antonova, V. V. Vasin, E. A. Pimonov, and F. J. Kuchuk, in Theory and Numerical Methods for Solving Inverse and Ill-Posed Problems: Abstracts of Youth Internat. Scientific School-Conference (Inst. Mat. Sib. Otdel. Ross. Akad. Nauk, Novosibirsk, 2009), pp. 13-14 [in Russian].

7. M. Hanke, Inverse Problems 13, 79 (1997).

8. V. V. Vasin and G. G. Skorik, J. Inverse Ill-Posed Probl. 18 (8), 855 (2010).

Translated by M. Deikalova 